\title{
Contexto da unidade de terapia intensiva: análise da produção científica da enfermagem
}

Intensive care unit context: analysis of nursing scientific production Contexto de la unidad de cuidados intensivos: análisis de la producción científica de enfermería

\section{Alcides Viana de Lima Neto ${ }^{I}$, Andréa Tayse de Lima Gomes ${ }^{I I}$, Cecília Olívia Paraguai de Oliveira Saraiva ${ }^{\mathrm{III}}$, Suzane Gomes de Medeiros ${ }^{\mathrm{IV}}$, Mayara Lima Barbosav ${ }^{\mathrm{V}}$, Viviane Euzébia Pereira Santos ${ }^{\mathrm{VI}}$}

Resumo: Objetivo: caracterizar as dissertações e teses disponíveis no Catálogo de Teses e Dissertações da Associação Brasileira de Enfermagem oriundas de pesquisas sobre o contexto de unidade de terapia intensiva. Método: pesquisa bibliométrica, desenvolvida a partir de dados extraídos por meio de um instrumento composto por indicadores coletados das dissertações e teses disponíveis no catálogo dos volumes XIX (2001) ao XXXIV (2014). Incluíram-se todas as dissertações e teses produzidas por enfermeiros, provenientes de pesquisa em unidades de terapia intensiva. Resultados: destacaram-se as pesquisas relacionadas aos cuidados e procedimentos de enfermagem (46; 18,9\%); carga de trabalho, estresse e dimensionamento de pessoal de enfermagem (28; $11,5 \%$ ) e sistematização da assistência e processo de enfermagem $(20 ; 8,2 \%)$, ambos na unidade de terapia intensiva. Conclusão: as pesquisas analisadas revelam um perfil no qual a maioria dos estudos apresentaram um delineamento descritivo-quantitativo e com foco nos cuidados e procedimentos realizados nas unidades de terapia intensiva.

Descritores: Unidade de Terapia Intensiva; Enfermagem; Pesquisa; Procedimentos Clínicos; Cuidados Críticos

Abstract: Objective: to characterize the dissertations and theses available in the Catalog of Theses and Dissertations of the Brazilian Nursing Association from research on the context of the intensive care unit. Method: bibliometric research, developed from data extracted through an instrument composed of indicators collected from

\footnotetext{
IEnfermeiro. Mestre em enfermagem. Universidade Federal do Rio Grande do Norte. Natal, RN, Brasil. E-mail: alcides.vln@gmail.com. ORCID: https://orcid.org/0000-0001-6191-9465.

${ }^{\text {II } E n f e r m e i r a . ~ M e s t r e ~ e m ~ e n f e r m a g e m . ~ U n i v e r s i d a d e ~ F e d e r a l ~ d o ~ R i o ~ G r a n d e ~ d o ~ N o r t e . ~ N a t a l, ~ R N, ~ B r a s i l . ~ E-m a i l: ~ a n d r e a . t l g o m e s @ g m a i l . c o m . ~}$ ORCID: https://orcid.org/0000-0003-0505-5783.

IIIEnfermeira. Doutora em enfermagem. Universidade Federal do Rio Grande do Norte. Natal, RN, Brasil. E-mail: cecilia_olivia@yahoo.com.br. ORCID: https://orcid.org/0000-0003-4225-5194.

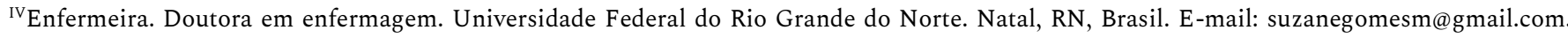
ORCID: https://orcid.org/0000-0002-4196-4557.

${ }^{\vee}$ Enfermeira. Doutora em enfermagem. Professora da Faculdade de Ciências Médicas do Centro Universitário UNIFACISA. Campina Grande, PB, Brasil. E-mail: mayaralimabarbosa@gmail.com. ORCID: https://orcid.org/0000-0002-8063-7903.

${ }^{\mathrm{VI}}$ Enfermeira. Doutora em enfermagem. Professora da Universidade Federal do Rio Grande do Norte. Natal, RN, Brasil. E-mail: vivianeepsantos@gmail.com. ORCID: https://orcid.org/0000-0001-8140-8320.
} 
dissertations and theses available in the catalog of volumes XIX (2001) to XXXIV (2014). All dissertations and theses produced by nurses from research in intensive care units were included. Results: research related to nursing care and procedures stood out $(46 ; 18.9 \%)$; workload, stress and sizing of nursing staff $(28 ; 11.5 \%)$ and systematization of nursing care and process $(20 ; 8.2 \%)$, both in the intensive care unit. Conclusion: The research analyzed reveals a profile in which most studies presented a descriptive-quantitative design and focused on the care and procedures performed in intensive care units.

Descriptors: Intensive Care Units; Nursing; Research; Critical Pathways; Critical Care

Resumen: Objetivo: caracterizar las disertaciones y tesis disponibles en el Catálogo de Tesis y Disertaciones de la Asociación Brasileña de Enfermería de investigaciones sobre el contexto de la unidad de cuidados intensivos. Método: investigación bibliométrica, desarrollada a partir de datos extraídos por un instrumento compuesto por indicadores recopilados de disertaciones y tesis disponibles en catálogo de los volúmenes XIX (2001) a XXXIV (2014). Incluyeron todas las disertaciones y tesis producidas por enfermeras originado de investigaciones en unidades de cuidados intensivos. Resultados: se destacó la investigación relacionada con atención y procedimientos de enfermería (46; 18,9\%); carga de trabajo, estrés y dimensionamiento del personal (28; $11.5 \%$ y sistematización de cuidados y procesos de enfermería $(20 ; 8.2 \%)$, ambos en la unidad de cuidados intensivos. Conclusión: La investigación revela un perfil en que la mayoría de los estudios presentaron un diseño descriptivocuantitativo y se centraron en atención y procedimientos realizados en unidades de cuidados intensivos.

Descriptores: Unidades de Cuidados Intensivos; Enfermería; Investigación; Vías Clínicas; Cuidados Críticos

\section{Introdução}

A enfermagem, considerada uma ciência, apresenta uma tendência contemporânea de aumento na produção de pesquisas que contribuem com a prática de cuidados baseada em evidências. Cada vez mais, demanda-se do enfermeiro, além das competências técnicas, a capacidade de compreender o contexto científico e desenvolver pesquisas nos diversos cenários da prática profissional. ${ }^{1}$

Por possuir um campo de saberes e conhecimentos específicos, a profissão evoluiu historicamente e conquistou espaços em áreas temáticas e linhas de pesquisa diferentes. Isso contribuiu com a qualificação de pesquisadores nesses campos, aptos a desempenharem atividades nos diferentes contextos que apresentam a inserção desses profissionais, além de qualificar o cuidado ofertado aos pacientes. $^{2}$

Assim, sabe-se que grande parte das pesquisas brasileiras são desenvolvidas nos programas de pós-graduação stricto sensu, que oferecem cursos de mestrado e de doutorado nas 
modalidades acadêmica e profissional. Ambos são acompanhados pela Fundação Coordenação de Aperfeiçoamento de Pessoal de Nível Superior (CAPES), órgão vinculado ao Ministério da Educação. Os cursos de mestrado e doutorado acadêmico apresentam um perfil direcionado a formação para a carreira acadêmica e a pesquisa. ${ }^{3}$ Já os mestrados e doutorados profissionais possibilitam capacitar para o exercício da prática avançada e transformadora de procedimentos para atenderem as demandas sociais, organizacionais ou profissionais e do mercado de trabalho. ${ }^{4}$ Esses últimos, ofertados em menor número, porém em expansão no Brasil.

Segundo dados da CAPES, na área de avaliação de enfermagem, existem 78 programas, distribuídos em todas as regiões brasileiras, com maior concentração nas regiões Sudeste e Nordeste, com 32 e 20 respectivamente, o que representa $66,67 \%$ do total. Nesses programas são desenvolvidos 116 cursos, dos quais, 52 são mestrados acadêmicos, 38 doutorados acadêmicos, 24 mestrados profissionais e 2 doutorados profissionais. ${ }^{5}$

Em relação às linhas de pesquisa, destacam-se: fundamentos teórico-filosóficos do cuidar em saúde e enfermagem, tecnologia em saúde e enfermagem, ética em saúde e enfermagem e história da enfermagem, processo de cuidar em saúde e enfermagem, saúde e qualidade de vida e no campo organizacional, políticas e práticas em saúde e enfermagem, políticas e práticas de educação e enfermagem, produção social e trabalho em saúde e enfermagem, gerenciamento dos serviços de saúde e de enfermagem e informação/comunicação em saúde e enfermagem. ${ }^{6}$

Dentre essas linhas, muitas pesquisas são desenvolvidas no ambiente das unidades de terapia intensiva (UTI), que é a área crítica destinada à internação de pacientes graves, que requerem atenção profissional especializada de forma contínua, materiais específicos e tecnologias necessárias ao diagnóstico, monitorização e terapia. ${ }^{7}$ A UTI, por apresentar uma complexidade diferente de outros setores, requer um dimensionamento diferenciado dos profissionais de enfermagem, assim como, capacitação para uma tomada de decisão rápida e 
Contexto da unidade de terapia intensiva: análise da produção científica da enfermagem | 4

imediata diante de situações que demandam um raciocínio crítico e reflexivo para a resolução de determinados problemas.

Portanto, é importante o desenvolvimento de estudos que ofereçam um perfil do conhecimento produzido sobre o cuidado intensivo de enfermagem no âmbito da pós-graduação brasileira com a finalidade de identificar os principais problemas investigados, as evidências produzidas sobre a temática e as lacunas de pesquisa. Dessa maneira, podem ser oferecidos subsídios para o conhecimento dessa realidade, bem como, um embasamento para o desenvolvimento de diversas investigações.

Logo, o presente estudo objetivou caracterizar as dissertações e teses disponíveis no Catálogo de Teses e Dissertações da Associação Brasileira de Enfermagem oriundas de pesquisas sobre o contexto de unidade de terapia intensiva.

\section{Método}

Trata-se de uma pesquisa bibliométrica, desenvolvida a partir da análise das dissertações e teses disponíveis no Catálogo de Teses e Dissertações (CEPEn) dos volumes XIX (2001) ao XXXIV (2014). Escolheu-se esse período devido a disponibilidade dos catálogos dos resumos na página eletrônica da Associação Brasileira de Enfermagem (ABEn). Para a sua realização elaborou-se um protocolo de pesquisa estruturado em: tema; objetivo; questão norteadora; estratégias de busca; seleção dos estudos; coleta de dados; avaliação crítica dos estudos e síntese de dados. Não foram utilizadas palavras-chave, uma vez que foi realizada a leitura na íntegra de todos os volumes do catálogo para a seleção das teses e dissertações.

A busca foi realizada norteada pela seguinte questão: quais as características das dissertações e teses disponíveis no CEPEn da ABEn provenientes de pesquisas em unidades de terapia intensiva? Para a operacionalização da mesma, adotou-se a estratégia de leitura inicial de todos os resumos das teses e dissertações disponíveis na versão online do catálogo. Com base 
nessa leitura inicial, elaborou-se uma lista no software Microsoft Excel ${ }^{\circledR}$ versão 2010 contendo o título, autores, instituição de ensino superior (IES) e ano de defesa. Essa lista serviu para nortear a busca futura dos documentos na íntegra no Catálogo de Teses e Dissertações da CAPES. Ressalta-se que a busca foi realizada por dois pesquisadores independentes, ambos alunos do curso de mestrado acadêmico em enfermagem da Universidade Federal do Rio Grande do Norte.

Incluíram-se todas as dissertações e teses, componentes do CEPEn da ABEn, produzidas por enfermeiros, provenientes de pesquisas em unidades de terapia intensiva. Excluíram-se as dissertações e teses com resumos incompletos, as que não responderam aos indicadores de coleta em sua totalidade; dissertações e teses com texto completo indisponível para download e as que foram construídas a partir de revisões de literatura. Realça-se que não houve discordância durante todo o processo de busca e seleção dos documentos.

Após a análise de cada tese e dissertação na íntegra, os seguintes indicadores foram coletados: nível acadêmico (mestrado ou doutorado); instituição de ensino; ano de publicação; local de desenvolvimento do estudo (região e Estado); objeto de estudo; desenho metodológico: tipo de estudo, abordagem (quantitativa, qualitativa ou mista); nível de evidência segundo o Joana Briggs Institute: I - estudos experimentais; revisões sistemáticas de estudos experimentais; II - estudos quase-experimentais; III - estudos observacionais; IV - estudos descritivos; V - opiniões e consensos de especialistas; tipo de UTI pesquisada (adulto, neonatal, pediátrica, pediátrica mista); sujeitos da pesquisa (pacientes; enfermeiros; técnicos de enfermagem; auxiliares de enfermagem; equipe de enfermagem; outros profissionais de saúde; familiares dos pacientes; outros - especificar). ${ }^{8}$

Para a análise dos dados, que foi realizada no mês de junho de 2017, utilizou-se categorização temática e estatística descritiva simples. A Figura 1 apresenta as etapas de seleção, análise e amostragem final dos documentos que integraram o estudo. 
Contexto da unidade de terapia intensiva: análise da produção científica da enfermagem | 6

Figura 1 - Fluxo do processo de seleção e análise dos documentos da pesquisa, 2018.

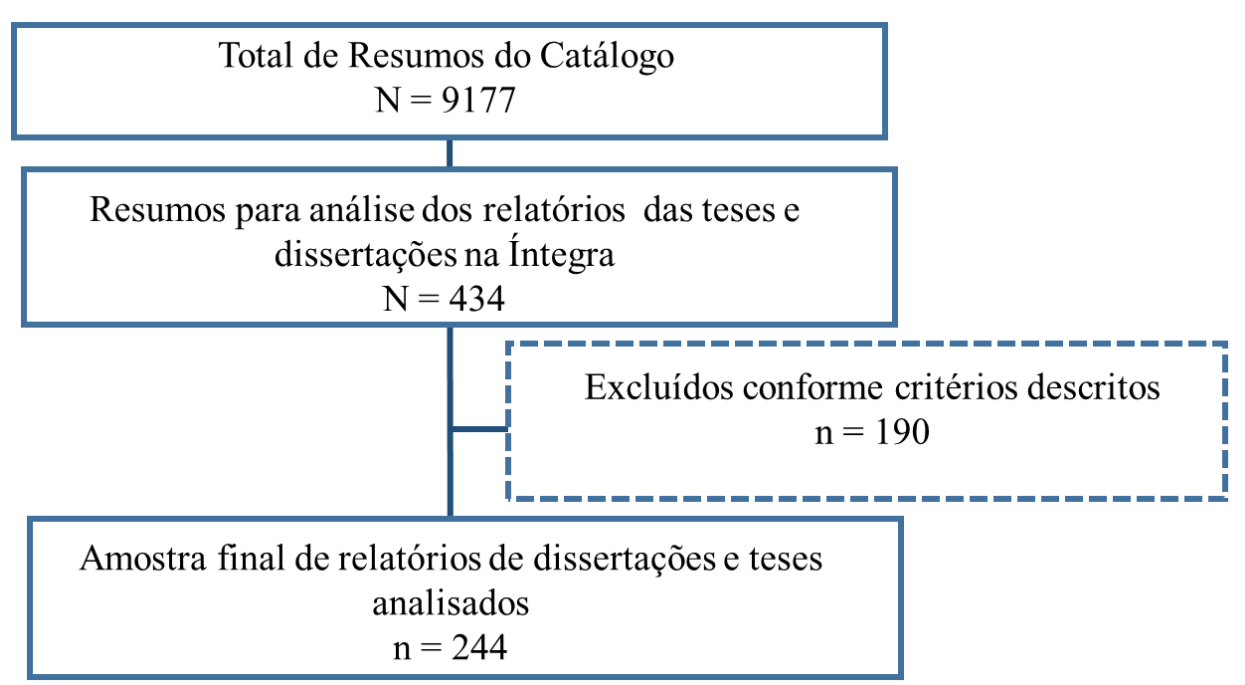

\section{Resultados}

No geral, o número de documentos teve um comportamento de distribuição irregular no período analisado, em números absolutos, nos seus respectivos volumes, de: 20 (volume XXXIV - 2014), 24 (volume XXXII - 2013), 46 (volume XXXI - 2012), 34 (volume XXX - 2011), 19 (volume XXIX - 2010), 21 (volume XXVIII - 2009), 19 (volume XXVII - 2008), 10 (volume XXVI - 2007), 28 (volume XXV - 2006), 9 (volume XXIV - 2005), 2 (volume XXIII - 2004), 6 (volume XXII - 2003), 2 (volume XXI - 2º semestre de 2002), 1 (volume XX - 1º semestre de 2002), 3 (volume XIX - 2001). Apresentaram destaque os anos de 2006, 2011 e 2012, com o maior número de estudos.

A Tabela 1 apresenta as universidades e o quantitativo de estudos realizados nos seus respectivos programas de pós-graduação. Percebeu-se que a maioria foi realizada nas Universidades de São Paulo e Federal de Santa Catarina.

Tabela 1 - Dissertações e teses provenientes de pesquisas em universidades brasileiras, 2018.

\begin{tabular}{lc} 
Instituição de Ensino Superior & $\mathbf{n}(\mathbf{\%})$ \\
\hline Universidade de São Paulo & $86(35,3 \%)$ \\
Universidade Federal de Santa Catarina & $40(16,3 \%)$ \\
Universidade Federal de Minas Gerais & $27(11,1 \%)$ \\
Universidade Federal do Rio de Janeiro & $16(6,6 \%)$
\end{tabular}


7 | Lima Neto AV, Gomes ATL, Saraiva COPO, Medeiros SG, Barbosa ML, Santos VEP

$\begin{array}{lc}\text { Universidade Federal da Bahia } & 14(5,7 \%) \\ \text { Universidade Federal do Ceará } & 8(3,3 \%) \\ \text { Universidade Federal de Goiás } & 7(2,9 \%) \\ \text { Universidade Federal do Rio Grande do Norte } & 5(2,1 \%) \\ \text { Universidade Federal do Paraná } & 5(2,1 \%) \\ \text { Universidade Federal do Rio Grande } & 5(2,1 \%) \\ \text { Universidade Federal da Paraíba } & 5(2,1 \%) \\ \text { Universidade Federal do Estado do Rio de Janeiro } & 4(1,6 \%) \\ \text { Universidade Federal do Rio Grande do Sul } & 3(1,2 \%) \\ \text { Universidade Federal de Santa Maria } & 3(1,2 \%) \\ \text { Universidade de Campinas } & 3(1,2 \%) \\ \text { Universidade de Brasília } & 3(1,2 \%) \\ \text { Universidade Federal de Alagoas } & 2(0,8 \%) \\ \text { Universidade Estadual de Maringá } & 2(0,8 \%) \\ \text { Universidade Estadual de Londrina } & 2(0,8 \%) \\ \text { Universidade Federal de São Paulo } & 1(0,4 \%) \\ \text { Universidade Guarulhos } & 1(0,4 \%) \\ \text { Universidade Federal do Maranhão } & 1(0,4 \%) \\ \text { Universidade Federal do Espírito Santo } & 1(0,4 \%) \\ \text { Total } & \mathbf{2 4 4 ( \mathbf { 1 0 0 } \% )}\end{array}$

Em relação ao nível acadêmico, a maioria foi proveniente de cursos de mestrado (204; 83,6\%), seguida pelas produções a nível de doutorado $(40 ; 16,4 \%)$. Quanto a caracterização segundo o local onde foi realizada a pesquisa, a Figura 2 apresenta a distribuição por Unidade de Federação (UF). Percebeu-se uma concentração de estudos na região Sudeste, com mais da metade $(127 ; 52,0 \%)$, seguida pelas regiões Sul $(58 ; 23,8 \%)$ e Nordeste $(36 ; 14,8 \%)$. A região que apresentou o menor percentual foi a Centro-Oeste $(11 ; 4,5 \%)$. Nenhum dos estudos analisados foi realizado na região Norte e alguns não citaram o local de desenvolvimento $(12 ; 4,9 \%)$.

Ressalta-se que os dados apresentados na Figura 2 dizem respeito ao local onde os dados das pesquisas foram coletados, o que pode diferenciar da localização geográfica da universidade na qual o curso é ofertado. 
Contexto da unidade de terapia intensiva: análise da produção científica da enfermagem $\mid 8$

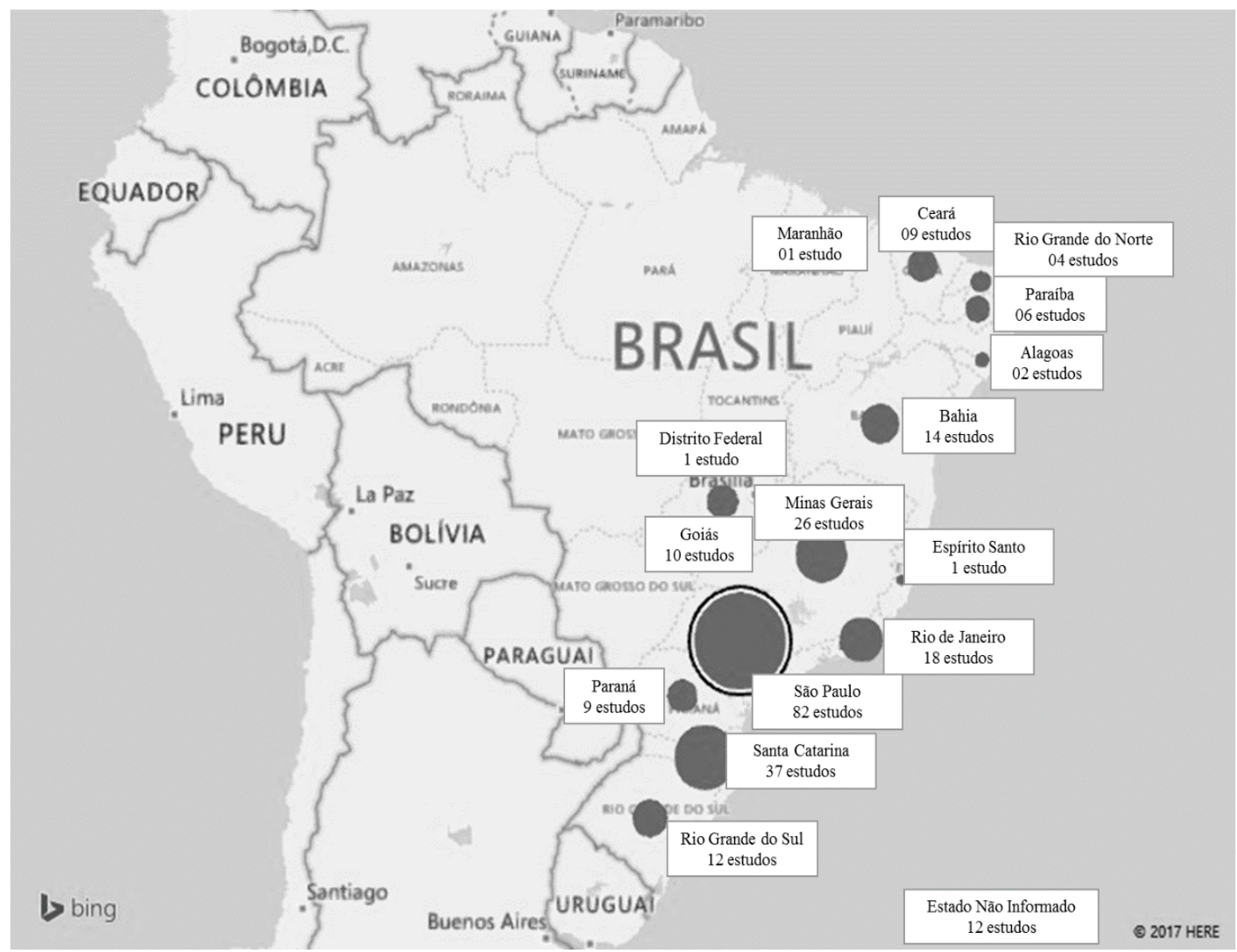

Figura 2 - Quantitativo de estudos por Unidade de Federação brasileira, 2018.

Os objetos de estudo das teses e dissertações estiveram relacionados, principalmente a: cuidados e procedimentos de enfermagem na UTI (46; 18,9\%); carga de trabalho, estresse e dimensionamento de pessoal de enfermagem (28; 11,5\%); sistematização da assistência e processo de enfermagem na UTI (20; $8,2 \%)$; sentimentos e vivência dos familiares de pacientes internados na UTI (19; 7,8\%); não informado (15; 6,2\%); processo de trabalho da enfermagem na UTI (13; 5,3\%); qualidade, cultura e segurança do paciente (13; 5,3\%); infecções relacionadas a assistência à saúde $(12 ; 4,9 \%)$; saúde do trabalhador, qualidade de vida dos profissionais, riscos ocupacionais e biossegurança (11; 4,5\%); lesões por pressão e outras lesões em pacientes internados na UTI $(10 ; 4,1 \%)$.

Quanto ao desenho metodológico, predominaram os estudos do tipo descritivos (202; $82,9 \%)$, seguidos dos estudos observacionais $(11 ; 4,5 \%)$. No que diz respeito ao delineamento, 
estiveram em realce os estudos quantitativos $(133 ; 54,5 \%)$ e qualitativos $(85 ; 34,8 \%)$. Os demais foram mistos $(20 ; 8,2 \%)$ e alguns não informaram $(6 ; 2,5 \%)$.

A partir dos tipos, os estudos foram classificados segundo os critérios do $\mathrm{JBI}^{(8)}$ nos seguintes níveis de evidência: I (3; 1,2\%); II (5; 2,1\%); III (15; 6,2\%); IV (221; 90,5\%). A abordagem predominante foi a quantitativa $(133 ; 54,5 \%)$, seguida pela qualitativa $(85 ; 34,8 \%)$ e mista $(20$; $8,2 \%)$. Seis estudos (2,5\%) não informaram a abordagem no método.

Em relação ao tipo de UTI nas quais foram realizadas as pesquisas, identificou-se um maior percentual em UTI adulto (165; 67,6\%). Tiveram ainda estudos realizados em UTI neonatal (46; 18,9\%), UTI pediátrica (14; 5,7\%), alguns realizados simultaneamente em UTI adulto e pediátrica (6; $2,5 \%)$, adulto e neonatal $(1 ; 0,4 \%)$, adulto, pediátrica e neonatal $(1 ; 0,4 \%)$, pediátrica e neonatal $(1 ;$ 0,4\%) e em UTI móvel $(1 ; 0,4 \%)$. Nove estudos (3,7\%) não informaram o tipo de UTI pesquisada.

Os sujeitos da pesquisa foram categorizados e distribuídos, conforme apresentado nos estudos. A maioria deles foi realizada com pacientes $(84 ; 34,4 \%)$, enfermeiros $(54 ; 22,1 \%)$, equipe de enfermagem - não especificou se o público foram enfermeiros ou técnicos $(46 ; 18,9 \%)$ e familiares dos pacientes $(16 ; 6,6 \%)$ que juntos, totalizam $82,0 \%$ dos sujeitos dos estudos.

\section{Discussão}

Os documentos analisados representam uma amostra de apenas 2,66\% de todas as pesquisas realizadas nos programas de pós-graduação de enfermagem brasileiros. Esse número pode ser considerado baixo, diante da complexidade que envolve o processo de cuidar do paciente nesses ambientes. É notório que o trabalho na UTI objetiva um atendimento qualificado e ininterrupto às pessoas que necessitam de monitoramento intensivo, devido às suas condições clínicas bem como aquelas que apresentam risco de morrer. ${ }^{9}$

Diante dessa expressividade, destaca-se a concentração de pesquisas nas regiões Sudeste, Sul e Nordeste no período analisado. Esse fato pode ter ocorrido devido ao maior número de leitos para 
Contexto da unidade de terapia intensiva: análise da produção científica da enfermagem | 10

tratamento intensivo estar localizado nessas regiões, segundo dados de uma pesquisa realizada pelo Conselho Federal de Medicina. ${ }^{10}$ Ressalta-se que nessas regiões também está localizada a maioria dos cursos de pós-graduação em nível de mestrado e doutorado em enfermagem. ${ }^{5}$

No que diz respeito aos objetos de estudo, destaca-se a importância deles ao contribuírem para a qualificação do cuidado nos diferentes cenários de prática do profissional. Cuidados esses, que também podem envolver a realização de procedimentos técnicos e demandam uma carga de responsabilidades para a sua execução. ${ }^{11}$ Esses objetos foram os de maior relevância, uma vez que apresentaram predominância em número de produções.

Observou-se ainda um número significativo de estudos que abordam a carga de trabalho, estresse e o dimensionamento de pessoal de enfermagem. Esses três pontos estão relacionados e interferem na qualidade de vida dos trabalhadores e são objetos de estudos internacionais ${ }^{12-13}$ e nacionais. $^{14-15}$

Assim, uma pesquisa realizada em oito UTIs de um hospital público de São Paulo revelou que os profissionais de enfermagem consideram os fatores desencadeadores do estresse no ambiente de trabalho um desafio. É posto que esse também é desafiador para o contexto organizacional, uma vez que o estresse pode implicar em comprometimento na qualidade assistencial. ${ }^{15}$

Estudo cita que no atual contexto, muitos profissionais de enfermagem passam por situações que geram carga de trabalho elevada e pressão nos ambientes das UTIs, o que pode interferir diretamente na vida pessoal e no processo de cuidar. ${ }^{16}$ Tais situações podem estar relacionadas à exposição dos profissionais e dos pacientes às situações de risco, manuseio de muitos recursos tecnológicos, convivência com o processo de morte e morrer, ansiedade, dentre outros. $^{9}$ Portanto, se torna indispensável considerar os fatores que interferem na vida do profissional e ações que promovam a saúde do trabalhador de enfermagem. ${ }^{16}$

Em relação a caracterização metodológica, percebeu-se que foi comum a presença de estudos descritivos, quantitativos e com a maior classificação no nível de evidência IV. Esse 
11 | Lima Neto AV, Gomes ATL, Saraiva COPO, Medeiros SG, Barbosa ML, Santos VEP

resultado também foi encontrado em outra investigação que analisou produções da pósgraduação em enfermagem brasileira. ${ }^{17}$ Embora esse tipo de pesquisa apresente a sua importância, mais especificamente em analisar e explicar o que acontece na prática profissional, ainda é necessário gerar conhecimento dessas situações, o que demanda o desenvolvimento de estudos em outros níveis de evidência. ${ }^{18}$

Ao se tratar especificamente dos tipos de UTI, nas quais foram realizadas as pesquisas, destacou-se a expressividade do cenário da terapia intensiva adulto, que é a destinada à assistência de pacientes com idade igual ou superior a 18 anos, e que pode também admitir pacientes de 15 a 17 anos, se definido nas normas da instituição. ${ }^{7}$

Ressalta-se que são raros os estudos que tratam do perfil dessas unidades no Brasil. Um projeto desenvolvido pela Associação de Medicina Intensiva Brasileira (AMIB) em parceria com a Epimed Solutions, intitulado UTIs brasileiras, objetivou caracterizar o perfil epidemiológico das UTIs brasileiras e compartilhar informações que possam ser úteis para orientar políticas de saúde e estratégias para melhorar o cuidado dos pacientes críticos no referido país. Segundo dados do projeto, 621 unidades aderiram. Porém, não foi citada classificação por tipo de unidade segundo a faixa etária dos pacientes. ${ }^{19}$

O público-alvo das pesquisas foi, em sua maioria, pacientes admitidos para tratamento, seguido por enfermeiros e equipe de enfermagem de forma geral. O técnico de enfermagem foi sujeito exclusivo de apenas uma das pesquisas, o que representa uma incipiência de investigações nesse cenário a partir desses profissionais.

Dessa maneira, além dos estudos menos frequentes evidenciados, considera-se que é necessário o desenvolvimento de pesquisas com um maior aprofundamento das respostas fisiológicas do paciente às intervenções de enfermagem no cuidado intensivo, uma vez que foram incipientes. Sugere-se também um olhar para os temas prioritários da Agenda de Prioridades de Pesquisa do Ministério da Saúde, ${ }^{20}$ cujos objetos de investigação podem se 
Contexto da unidade de terapia intensiva: análise da produção científica da enfermagem | 12

aplicar ao contexto da terapia intensiva, como o desenvolvimento de tecnologias e inovação em saúde e saúde materno-infantil e do idoso.

Ademais, como limitação do estudo, destaca-se a exclusão de 190 pesquisas, o que representa 39,63\% dos resumos selecionados para análise dos relatórios das teses e dissertações na íntegra, devido os documentos não estarem disponíveis para download. Esse número é expressivo, e pode ter influenciado os resultados aqui apresentados.

\section{Conclusão}

O estudo possibilitou caracterizar as teses e dissertações produzidas por enfermeiros que versam sobre temas de interesse no contexto da terapia intensiva. Apresentou um perfil no qual a maioria das pesquisas foram realizadas nas regiões Sudeste, Sul e Nordeste, com delineamento descritivo e com foco nos cuidados e procedimentos realizados na UTI.

Assim, percebeu-se que os temas em destaque apresentam relevância contemporânea para a enfermagem por estarem relacionados aos procedimentos executados por essa equipe, carga de trabalho, estresse e dimensionamento, além da sistematização da assistência, ambos no contexto da terapia intensiva.

Apesar disso, recomenda-se o desenvolvimento de mais pesquisas por enfermeiros no cenário da terapia intensiva com níveis de evidência I, II e III, o que pode contribuir ainda mais com a qualificação dos cuidados ofertados ao paciente em tratamento intensivo, além de outros processos que envolvem o cotidiano do trabalho da enfermagem. Sugere-se também que sejam realizadas novas pesquisas semelhantes a esta para que o perfil dos estudos desenvolvidos nos anos seguintes a este seja traçado. 


\section{Referências}

1. Cassiani SHB, Bassalobre-Garcia A, Reveiz L. Universal Access to Health and Universal Health Coverage: identification of nursing research priorities in Latin America. Rev Latinoam Enferm [Internet]. 2015 [acesso em 2017 jul 05];23(6):1195-208. Disponível em: http://www.scielo.br/pdf/rlae/v23n6/0104-1169-rlae-10752667.pdf

2. Erdmann AL, Peiter CC, Lanzon GMM. Grupos de pesquisa em enfermagem no Brasil: comparação dos perfis de 2006 e 2016. Rev Gaúch Enferm [Internet]. 2017 [acesso em 2020 jan 09];38(2):e69051. Disponível em: https://www.seer.ufrgs.br/RevistaGauchadeEnfermagem/article/view/69051/42008

3. Figueredo WN, Laitano AC, Santos VPFA, Dias ACS, Silva GTR, Teixeira GAS. Formação didáticopedagógica na pós-graduação stricto sensu em Ciências da Saúde nas Universidades Federais do Nordeste do Brasil. Acta Paul Enferm [Internet]. 2017 [acesso em 2020 jan 07];30(5):497-503. Disponível em: https://pdfs.semanticscholar.org/b3b9/9ab135ae1e10286c358f40ab1661e446aa8a.pdf

4. Brasil. Ministério da Educação. Portaria nº 388, de 23 de março de 2017. Dispõe sobre o mestrado e doutorado profissional no âmbito da pós-graduação stricto sensu [Internet]. Brasília (DF): Ministério da Educação; 2017 [acesso em 2020 jan 07]. Disponível em: http://www.capes.gov.br/images/stories/download/legislacao/24032017PORTARIA-No-389-DE-23-DE-MARCO-DE-2017.pdf

5. Ministério da Educação (BR). Coordenação de Aperfeiçoamento de Pessoal de Nível Superior (CAPES). Plataforma Sucupira. Cursos avaliados e reconhecidos [Internet]. Brasília (DF): Ministério da Educação; 2020 [acesso em 2020 jan 07]. Disponível em: https://sucupira.capes.gov.br/sucupira/public/consultas/coleta/programa/quantitativos/quantitativoAreaConheci mento.jsf?areaAvaliacao $=20$

6. Associação Brasileira de Enfermagem; Centro de Estudos e Pesquisas em Enfermagem (CEPEn). Centro de Estudos e Pesquisas em Enfermagem. Brasília (DF): ABEn; 2015 [acesso em 2017 ago 28]. Disponível em: http://www.abennacional.org.br/centrodememoria/apresentacaocepen.htm

7. Ministério da Saúde (BR). Agência Nacional de Vigilância Sanitária (ANVISA). Resolução-RDC nº 7, de 24 de fevereiro de 2010. Dispõe sobre os requisitos mínimos para funcionamento de Unidades de Terapia Intensiva e dá outras providências [Internet]. Brasília (DF); 2010 [acesso em 2017 set 03]. Disponível em: http://bvsms.saude.gov.br/bvs/saudelegis/anvisa/2010/res0007_24_02_2010.html

8. Joanna Briggs Institute. The JBI Approach. Adelaide (Austrália); 2014 [acesso em 2017 ago 01]. Disponível em: https://joannabriggs.org/jbi-approach.html\#tabbed-nav=Levels-of-Evidence

9. Nascimento KC, Erdmann AL, Nascimento ERP, Santos JLG. Representações do cuidado no limiar da vida por profissionais de uma Unidade de Terapia Intensiva móvel. Enferm Foco [Internet]. 2016 [acesso em 2017 nov 15];7(1):71-5. Disponível em: http://revista.cofen.gov.br/index.php/enfermagem/article/view/671 
Contexto da unidade de terapia intensiva: análise da produção científica da enfermagem | 14

10. Ministério da Saúde (BR). Conselho Federal de Medicina. Medicina intensiva no Brasil [Internet]. Brasília (DF): Conselho Federal de Medicina; 2016 [acesso em 2018 jan 08]. Disponível em: https://portal.cfm.org.br/images/PDF/leitosuti_porregiao.pdf

11. Sousa MSM, Vieira LN, Carvalho SB, Monte NL. Os cuidados de enfermagem com os recém-nascidos na UTI. Rev Saúde Foco [Internet]. 2016 [acesso em 2018 jan 18];3(1):94-106. Disponível em: http://www4.fsanet.com.br/revista/index.php/saudeemfoco/article/view/923/1004

12. Mohammadi M, Peyrovi H, Mahmood M. The relationship between professional quality of life and caring ability in critical care nurses. Dimens Crit Care Nurs [Internet]. 2017 [acesso em 2020 jan 09];36(5):273-7. Disponível em: https://journals.lww.com/dccnjournal/Abstract/2017/09000/The_Relationship_Between_Professional_Qua lity_of.2.aspx

13. Abbasi M, Zakerian A, Akbarzade A, Dinarvand N, Ghaljahi M, Poursadeghiyan M, et al. Investigation of the relationship between work ability and work-related quality of life in nurses. Iran J Public Health [Internet]. 2017 [acesso em 2020 jan 09];46(10):1404-12. Disponível em: https://www.ncbi.nlm.nih.gov/pmc/articles/PMC5750353/pdf/IJPH-46-1404.pdf

14. Reich R, Vieira DFVB, Lima LB, Rabelo-Silva ER. Carga de trabalho em unidade coronariana segundo o Nursing Activities Score. Rev gaúcha enferm [Internet]. 2015 [acesso em 2017 out 18];36(3):2835. Disponível em: http://www.seer.ufrgs.br/RevistaGauchadeEnfermagem/article/view/51367

15. Andolhe R, Barbosa RL, Oliveira EM, Costa ALS, Padilha KG. Estresse, coping e Burnout da equipe de enfermagem de Unidades de Terapia Intensiva: fatores associados. Rev Esc Enferm USP [Internet]; 2015 [acesso em 2017 nov 28].49(N Esp):58-64. Disponível em: http://www.scielo.br/pdf/reeusp/v49nspe/1980-220X-reeusp-49-spe-0058.pdf

16. Alves EF. O cuidador de enfermagem e o cuidar em uma Unidade de Terapia Intensiva. J Health Sci [Internet]. 2015 [acesso em 2017 nov 30];15(2):115-22. Disponível em: http://www.pgsskroton.com.br/seer/index.php/JHealthSci/article/view/707/672

17. Meneses AS, Sanna MC. Research methods used in the production of knowledge on nursing administration. Rev Eletrônica Enferm [Internet]. 2016 [acesso em 2018 jan 02];17(4):1-11. Disponível em: https://www.revistas.ufg.br/fen/article/view/34648/20692

18. Scochi CGS, Munari DB, Padovani NA, Padilha KG, Lima MADS, Ferreira MA, et al. Doctorado en Enfermería en Brasil: formación en investigación y producción de tesis. Rev Latinoam Enferm [Internet]. 2015 [acesso em 2017 nov 30];23(3):387-94. Disponível em: http://www.scielo.br/pdf/rlae/v23n3/es_01041169-rlae-23-03-00387.pdf 
15 | Lima Neto AV, Gomes ATL, Saraiva COPO, Medeiros SG, Barbosa ML, Santos VEP

19. Epimed Solutions. UTIs Brasileiras. Registro Nacional de Terapia Intensiva. Perfil das Unidades de Terapia Intensiva. Rio de Janeiro; 2017 [acesso em 2018 jan 25]. Disponível em: http://www.utisbrasileiras.com.br/

20. Ministério da Saúde (BR). Agenda de Prioridades de Pesquisa do Ministério da Saúde [Internet]. Brasília (DF): Ministério da Saúde; 2018 [acesso em 2019 nov 01]. Disponível em: http://bvsms.saude.gov.br/bvs/publicacoes/agenda_prioridades_pesquisa_ms.pdf

\section{Autor correspondente}

Alcides Viana de Lima Neto

E-mail: alcides.vln@gmail.com

Endereço: Campus Universitário da UFRN, Departamento de Enfermagem, Av. Senador Salgado Filho, 3000, Candelária, Natal, RN.

CEP: 59064-741

\section{Contribuições de Autoria}

\section{1 - Alcides Viana de Lima Neto}

Concepção e planejamento do projeto de pesquisa, obtenção, análise e interpretação dos dados, redação e revisão crítica.

\section{2 - Andréa Tayse de Lima Gomes}

Obtenção ou análise e interpretação dos dados, redação e revisão crítica.

\section{3 - Cecília Olívia Paraguai de Oliveira Saraiva}

Obtenção ou análise e interpretação dos dados, redação e revisão crítica.

\section{4 - Suzane Gomes de Medeiros}

Obtenção ou análise e interpretação dos dados, redação e revisão crítica.

\section{5 - Mayara Lima Barbosa}

Obtenção ou análise e interpretação dos dados, redação e revisão crítica.

\section{6 - Viviane Euzébia Pereira Santos}

Concepção e planejamento do projeto de pesquisa, obtenção, análise e interpretação dos dados, redação e revisão crítica. 
Contexto da unidade de terapia intensiva: análise da produção científica da enfermagem | 16

\section{Como citar este artigo}

Lima Neto AV, Gomes ATL, Saraiva COPO, Medeiros SG, Barbosa ML, Santos VEP. Contexto da unidade de terapia intensiva: análise da produção científica da enfermagem. Rev. Enferm. UFSM. 2020 [Acesso em: Anos Mês Dia]; vol.10 e: 1-16. DOI:https://doi.org/10.5902/2179769234846 